\title{
Histopathological Examination and Literature Review of Orthokeratinized Odontogenic Cyst
}

\author{
Hirotoshi Iwai ${ }^{*}$, Takehiro Suzuki², Tomoko Suzuki², Juri Muramori², Hiroyuki Seto², \\ Mitsuko Nakayama², Tadahiko Utsunomiya' ${ }^{2}$, Masaaki Suemitsu², Kayo Kuyama², \\ Satoshi Hirayama ${ }^{1}$
}

${ }^{1}$ Department of Operative Dentistry, Nihon University School of Dentistry at Matsudo, Chiba, Japan

${ }^{2}$ Department of Pathology, Nihon University School of Dentistry at Matsudo, Chiba, Japan

Email: ^iwai.hirotoshi@nihon-u.ac.jp

How to cite this paper: Iwai, H., Suzuki, T., Suzuki, T., Muramori, J., Seto, H., Nakayama, M., Utsunomiya, T., Suemitsu, M., Kuyama, K. and Hirayama, S. (2019) Histopathological Examination and Literature Review of Orthokeratinized Odontogenic Cyst. Open Journal of Stomatology, 9, 125-135.

https://doi.org/10.4236/ojst.2019.95013

Received: April 22, 2019

Accepted: May 21, 2019

Published: May 24, 2019

Copyright ( 2019 by author(s) and Scientific Research Publishing Inc. This work is licensed under the Creative Commons Attribution International License (CC BY 4.0).

http://creativecommons.org/licenses/by/4.0/ (c) (i) Open Access

\begin{abstract}
Background: With transitions in the disease concept of orthokeratinized odontogenic cyst (OOC), the pathogenesis and etiology have not been sufficiently elucidated. Objectives: OOC cases were reclassified and observed to understand the clinico-histopathological characteristics. In addition, literature review of OOC was performed to better organize the pathology. Materials and methods: Subjects with jawbone cysts lined by keratinized stratified squamous epithelium from 2005 to 2018 were reclassified, and clinico-histopathological findings were analyzed. Previous reports of OOC/orthokeratinized-type odontogenic keratocyst (OKC) from 1980 to 2019 were organized. Results: Five cases of OOC were diagnosed, representing $2.1 \%$ of odontogenic developmental cysts (total, 239 cases). Mean age was 37.6 years, with a female predominance. The mandibular molar area was the frequent site, and all cysts were solitary. Sixty percent involved an impacted tooth. Mean maximum diameter of the cyst was $2.2 \mathrm{~cm}$. Histopathologically, 4 cases were unilocular, and partial palisading of the basal layer and scattered epithelial islands were observed in 2 cases each and formation of daughter cysts was noted in 3 cases. Previous papers described that most were unilocular and related to impacted teeth. Mean age ranged between 20 and 40 years, and the mandibular molar region was dominant. Recurrence rates were low. Conclusion: OOC shows a different biological attitude to OKC and is closer to dentigerous cyst. Meanwhile, OOC shows a similar histology of epidermoid cysts accompanying the granular layer. We thus surmised that OCC represents an independent concept as an odontogenic developmental cyst.
\end{abstract}

\section{Keywords}

Orthokeratinized Odontogenic Cyst (OOC), Odontogenic Developmental 


\section{Introduction}

Discussion of the diagnosis and classification of jawbone cysts has been continuing since Robinson first described classification based on the occurrence of epithelial tissue [1]. Orthokeratinized odontogenic cyst (OOC) has been considered as a type of odontogenic keratocyst (OKC). This concept, independent of odontogenic developmental cyst, has been inherited by the WHO classification of Head and Neck Tumours (WHO) [2], because of the many single cystic cases, involving an impacted tooth in around half of the cases and a lower frequency of postoperative recurrence. OOC was therefore defined as an odontogenic developmental cyst relined by orthokeratinized stratified squamous epithelium with a granule layer [3].

In recent years, the disease definition of OOC has been clarified. As a result, previous studies may have included some cases that should not have been included cysts with orthokeratinized cells in part of the lining epithelium of the OKC. With the transitions in the disease concept of OOC, the level of elucidation of the pathogenesis and etiology is now inadequate. Accordingly, jawbone cysts lining with keratinized stratified squamous epithelium were selected and reclassified in terms of OOC cases in accordance with the WHO [3] to clarify clinical and histopathological characteristics. In addition, a review of previous case reports and original papers on OOC was performed to better organize the pathology.

\section{Materials and Methods}

The present study was conducted in two parts: clinico-pathological and literature review studies.

\subsection{Subjects}

Subjects with odontogenic developmental cyst lined by keratinized stratified squamous epithelium in the jawbone were selected from the data files of the Department of Pathology at Nihon University School of Dentistry at Matsudo for the period from April 2005 to March 2018. With respect to clinical findings, age, gender, sites of occurrence, presence of impacted teeth, cyst form and size, and prognosis were analyzed.

\subsection{Histopathological Analysis}

Surgical materials were fixed in 10\% neutral-buffered formalin solution according to the usual method and embedded in paraffin. Specimens were prepared with a thickness of $4 \mu \mathrm{m}$. Specimens were stained then stained with hematoxylin and eosin (HE) and the cyst wall structure was observed in detail. Observation 
and reclassification of all cysts was performed by 3 oral pathologists in a blinded manner. Cysts partially covered by parakeratinized stratified squamous epithelium were categorized as OKC [3].

\subsection{Review of Literature}

Previous reports of OOC or orthokeratinized-type OKC were identified from a search of MEDLINE (National center for biotechnology information) for the period from 1980 to 2019. All the literature that was extracted was analyzed.

\subsection{Compliance with Ethical Standards}

Informed consent was obtained from all individuals included in the study. All procedures performed in studies involving human participants were in accordance with the ethical standards of the Committee on Studies Involving Human Beings of Nihon University School of Dentistry at Matsudo (EC18-16-025-1) and with the 1964 Declaration of Helsinki and its later amendments or comparable ethical standards.

\section{Results}

\subsection{Comparison of $\mathrm{OKC}$ and $\mathrm{OOC}$}

Odontogenic developmental cysts lined by keratinized stratified squamous cells was composed of OKC and OOC. OKC was identified in 234 patients (mean age, 43.0 years; range, 8 - 90 years), comprising 133 males (56.8\%) and 101 females (43.2\%), with a male-to-female ratio of 1.3:1. The site of occurrence was maxillary in 54 cases $(23.1 \%)$, mandibular in $148(62.7 \%)$, both maxillary and mandibular in $3(1.3 \%)$ and unknown in 29 (12.9\%).

In the present study, the total number of cases of OOC was 5, representing $2.1 \%$ of the 239 odontogenic developmental cysts lined by keratinized stratified squamous cells. Mean age was 37.6 years (range, 21 - 50 years), with 1 male $(20.0 \%)$ and 4 females $(80.0 \%)$, and thus a male-to-female ratio of 1:4. There were no complications in all patients. The site of occurrence was the molar area of the mandible in 4 cases (80.0\%), and the anterior maxilla in $1(20.0 \%)$. All the cysts were solitary. No associated with basal cell nevus syndrome was observed. Three cases $(60.0 \%)$ involved an impacted tooth. Mean maximum diameter of the cyst was $2.2 \mathrm{~cm}$, and all cysts were $\leq 4 \mathrm{~cm}$ in diameter.

\subsection{Histopathological Findings}

Representative histopathological findings are shown in Figure 1. In all cases, the cystic wall was lined by orthokeratinized stratified squamous epithelium with the appearance of the granular layer, and the cyst cavity were filled with feathery squamous material resembling the stratum corneum (Figure 1(a)). Four cases were unilocular, and one case was multilocular (Figure 1(b), arrow). Concerning the lining epithelium, the surface of the cyst wall was flat in 3 cases and wavy in 2 (Figure 1(c), arrow). The thickness of the spinous layer varied depending 

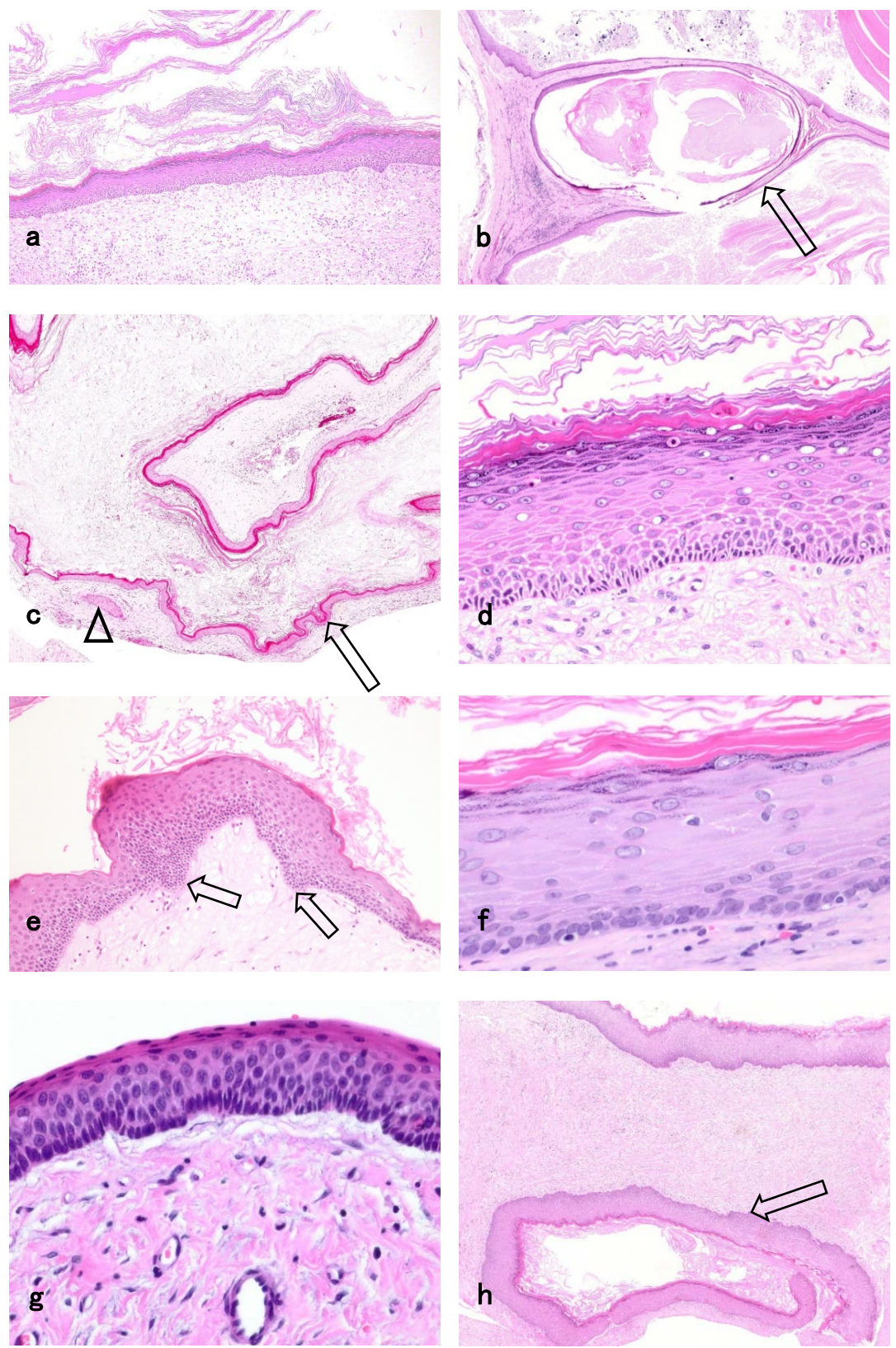

Figure 1. Representative histopathological findings of orthokeratinized odontogenic cyst (a-f, h) and odontogenic keratocyst (g) (H.E. staining). (a) The cyst cavity was filled with feathery squamous material resembling the stratum corneum $(\times 10)$; (b) Multilocular cyst was separated by thin cyst wall lined by hyperkeratotic epithelium (arrow, $\times 4$ ); (c) The wavy surface of the cyst wall (arrow) and epithelial island in the connective tissue (arrow head) $(\times 4)$. (d) $7-8$ layers in the thick wallined by orthokeratinized stratified squamous epithelium with the appearance of the granular layer $(\times 40)$. (e) Palisading and mild dropwise extension of the basal layer (arrow) was observed in the mixed type of cyst wall $(\times 10)$; (f) A morphology similar to stratified squamous epithelium of the basal layer $(\times 40)$; $(\mathrm{g})$ Greater concentration, swelling and palisading of the nucleus in odontogenic keratocyst ( $\times 40)$; (h) Formation of daughter cysts (arrow) was noted in the cyst wall $(\times 10)$.

on the case, with 2 - 3 layers in the thin type (Figure 1(c)), and 7 - 8 layers in the thick type (Figure 1(d)) and mixed type (Figure 1(e)). In terms of the mode of 
arrangement of the basallayer, partial palisading was observed in 2 cases (Figure 1(d), Figure 1(e)), and 4 cases showed a morphology similar to stratified squamous epithelium (Figure 1(f)). In cases of palisading, mild dropwise extension of the basal layer was observed (Figure 1(e), arrow). No cytologic atypia or mitosis was observed in the basal layer of any cases. Further comparison with the basal layer of OKC showed greater concentration of nuclear material, swelling and palisading of the nucleus (Figure 1(g)). Large peripheral nerves and blood vessels containing muscle fibers in the walls were not observed in the subepithelial connective tissue, but scattered epithelial islands were seen in 2 cases (Figure 1(c), arrowhead) and formation of daughter cysts was noted in 3 cases (Figure 1(h), arrow). The degree of inflammatory cell infiltration varied from mild to high. Granulation tissue with cholesterin crystals was seen in 2 cases. No skin appendages were apparent in the cyst wall of any cases.

\subsection{Review of Literatures of OCC}

Case reports of OCC are shown in Table 1 [4]-[11]. Ten cases were single, and

Table 1. Case reports of orthokeratinized odontogenic cyst.

\begin{tabular}{|c|c|c|c|c|c|c|c|c|c|c|c|c|}
\hline Refference No. & & & 4 & 5 & & 6 & & 7 & 8 & 9 & 10 & 11 \\
\hline Year & & & 2012 & 2013 & & 2013 & & 2014 & 2014 & 2015 & 2016 & 2017 \\
\hline \multirow[t]{2}{*}{ Cases } & Patinents (n) & & 1 & 1 & 1 & 1 & 1 & 1 & 1 & 1 & 1 & 1 \\
\hline & Cysts (n) & & 1 & 1 & 1 & 1 & 1 & 2 & 1 & 1 & 1 & 1 \\
\hline Average age & & & 40 & 25 & 73 & 27 & 61 & 23 & 30 & 46 & 50 & 41 \\
\hline Sex & & & female & female & femake & female & male & male & female & male & male & male \\
\hline \multirow[t]{5}{*}{ Location } & Lower & Molars - ramus & 1 & 0 & 1 & 1 & 1 & 2 & 1 & 1 & 0 & 0 \\
\hline & & Incisors - premolars & 0 & 1 & 0 & 0 & 0 & 0 & 0 & 0 & 0 & 0 \\
\hline & Upper & Molars & 0 & 0 & 0 & 0 & 0 & 0 & 0 & 0 & 0 & 0 \\
\hline & & Incisors - premolars & 0 & 0 & 0 & 0 & 0 & 0 & 0 & 0 & 1 & 1 \\
\hline & Unkwnon & & 0 & 0 & 0 & 0 & 0 & 0 & 0 & 0 & 0 & 0 \\
\hline Relation of BCNS ${ }^{*}$ & & & 0 & 0 & 0 & 0 & 0 & 0 & 0 & 0 & 0 & 0 \\
\hline \multirow[t]{2}{*}{ Cystic character } & Single & & 1 & 1 & 1 & 1 & 1 & 0 & 1 & 1 & 1 & 1 \\
\hline & Maltiple & & 0 & 0 & 0 & 0 & 0 & 1 & 0 & 0 & 0 & 0 \\
\hline \multirow[t]{2}{*}{ Form of cysts } & Unilocular & & 1 & 0 & 0 & 1 & 1 & 2 & 1 & 1 & 1 & 1 \\
\hline & Multilocular & & 0 & 1 & 1 & 0 & 0 & 0 & 0 & 0 & 0 & 0 \\
\hline \multirow[t]{2}{*}{$\begin{array}{c}\text { Relation of } \\
\text { impacted teeth }\end{array}$} & Present & & 1 & 0 & 0 & 1 & 1 & 2 & 1 & 0 & 1 & 0 \\
\hline & None & & 0 & 1 & 1 & 0 & 0 & 0 & 0 & 1 & 0 & 1 \\
\hline \multirow[t]{2}{*}{ Size of the cyst } & Over $4 \mathrm{~cm}$ & & 1 & 1 & - & - & - & - & - & 1 & 1 & 0 \\
\hline & Under $4 \mathrm{~cm}$ & & 0 & 0 & - & - & - & - & - & 0 & 0 & 1 \\
\hline Recurrence rate (\%) & & & 0 & 0 & 0 & 0 & 0 & 0 & 0 & 0 & 0 & 0 \\
\hline
\end{tabular}

*: Basal cell nevus syndrome. 
one involved bilateral cyst. Meanpatient age was 41.6 years (range, 23 - 73 years), with no apparent gender difference ( 5 men, 5 women). Site of occurrence was the mandibular molar area in 8 cases, the mandibular anterior premolar area in 1 , and the maxillary anterior premolar area in 2. Nine unilocular and 2 multilocular cysts were reported. Seven cases included an impacted tooth in the cyst cavity and 4 cases showed no tooth involvement. Cystsize was $>4 \mathrm{~cm}$ in 5 cases, $\leq 4 \mathrm{~cm}$ in 1 case, and unknown in 5 cases. No cases were associated with basal cell nevus syndrome, and no recurrences were observed.

Details of OCCs in the original papers are shown in Table 2 [12]-[18]. Iwabuchi et al. and Mizuda et al. described cases of multiple cysts, but most were solitary [14] [15]. Mean age ranged between 20 and 40 years, and gender differences were sometimes observed, depending on the report. Wright [12] described the cases involving the lower jaw was superior, and Crowley et al. [13] and MacDonald et al. [16] mentioned the advantage occurs in the posterior region. By contrast, many case reports [4] [6] [7] [8] [9] and original papers [12] [14] [15] [18] mentioned the site of occurrence as between the mandibular molar area and mandibular ramus.

Table 2. Original papers of orthokeratinized odontogenic cyst.

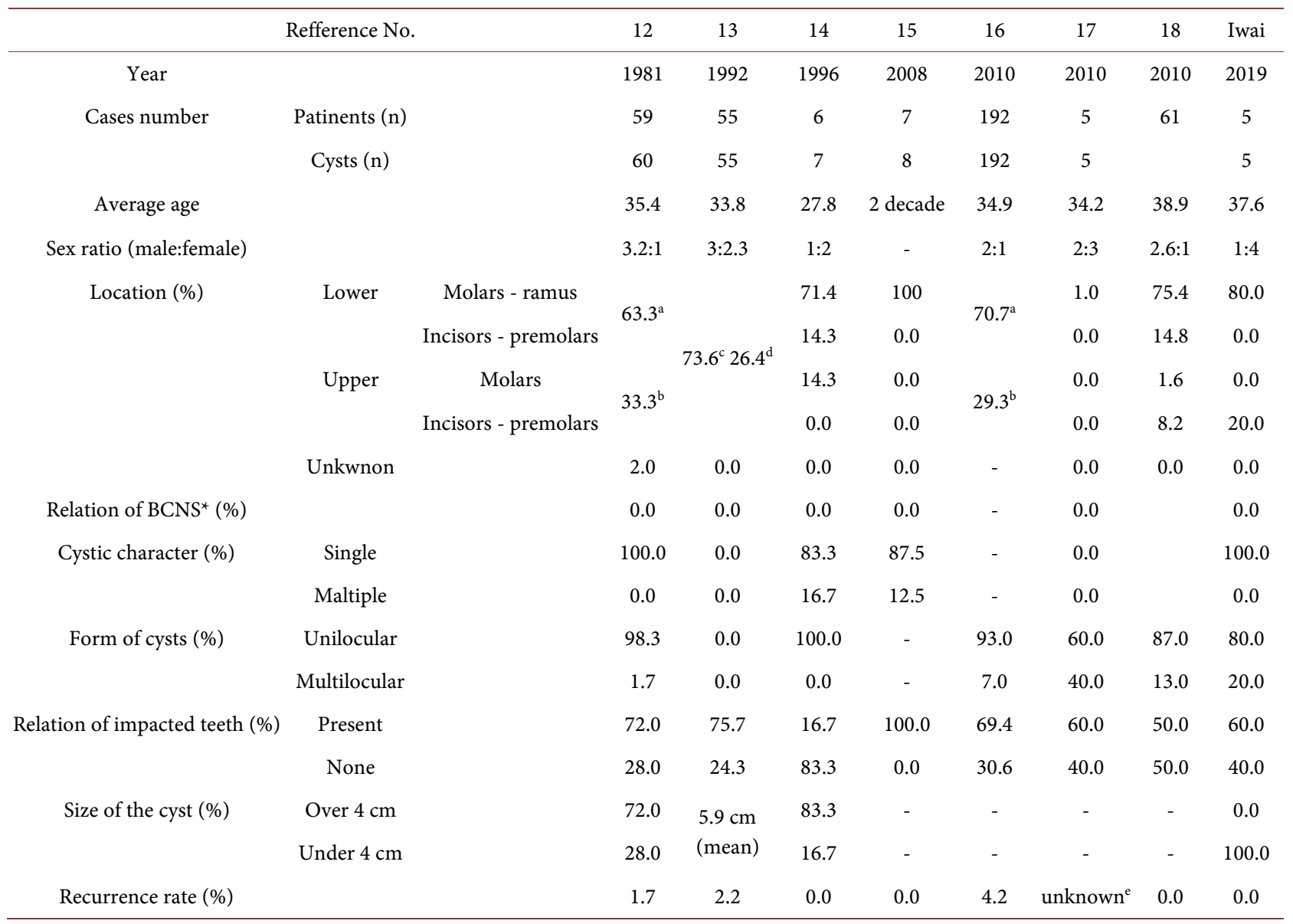

a: Unkwon of the location of the lower; b: Unknown of location of the upper; c: Molar area to ramus and tuberosity (unknown to maxilla or mandible); d: Anterior to premolar (unknown to maxilla or mandible); e: 4 cases were no recurrence and 1 case was no follow-up. 
Many reports described unilocular lesions, although Wright [12], MacDonald et al. [16] [17] and Dong et al. [18] reported multilocular cases. In terms of the relationship with teeth, only Iwabuchi et al. mentioned predominant non-involvement of impacted teeth [14]. No associations with basal cell nevus syndrome were observed. Recurrence rates were low, at $4.2 \%$ [16], 2.2\% [13], 1.7\% [12] and 0.0\% [14] [15] [18].

\section{Discussion}

Primordial cyst (PC) and OKC were classified as independent pathological entities in 2005 WHO classification of head and neck tumors [2]. Orthokeratinized-type PC has been suggested to be more appropriately named OOC [19] [20]. Further, PC, OKC and OOC are all described independently in the classification of Neville et al. [21]. Meanwhile, cases with mixed para-and orthokeratinized stratified squamous cell layers are treated as keratocystic odontogenic tumor (KCOT). Because a high rate of recurrence is seen with the mixed type [15], differentiation from OOC is important in planning treatment and follow-up.

Several theories have been advanced regarding the frequency of OOC, with only 9 cases reported over a period of 20 years in Malaysia [22]. On the other hand, Mizuta et al. [15] reported that in a study classifying KCOT/OKC based on the characteristics of the lining epithelium, 9\% of tumors represented orthokeratinized OKC, that is, OOC. Similar reports have mentioned OOC as representing $10 \%$ of KCOT/OKC [18], and about $1 \%$ of all odontogenic cysts [12] [13]. Iwabuchi et al. reported OOC as making up 19.4\% of KCOT/OKC, a substantially greater frequency than other reports [14]. A frequency of $2.1 \%$ was seen in the present study, and additional statistical information needs to be accumulated in the future incorporating the changes in disease concept.

OOC occurs over a broad age range, with a predilection for 30 - 40 years old, and has been described as showing a male predominance in previous studies [18] [24]. On the other hand, other reports have described 57\% of patients as in their 20s [14] [15], and previous reports [16] [24] with a mean age of 37.6 years and a female predominance. Average age in the present case was 42.9 years, and a slight maledominance was seen. Site of occurrence was reported as the lower jaw molar area to lower jaw branch in $71.4 \%$ by Iwabuchi et al. [14] and $100 \%$ by Mizuta et al. [15], respectively. Site of occurrence in the present study was the mandibularmolar area in $80.0 \%$. Both theories have been described in terms of the presence or absence of impacted teeth [4]-[11]. Although many previous reports have involved impacted teeth [12]-[18], Iwabuchi et al. reported a lower frequency of $16.8 \%$ [14]. In the present study, an impacted tooth component was present in $60.0 \%$ of all cases.

Cyst shape was often described as unilocular, although Wright [12] and MacDonald et al. [16] [17] have reported multilocular cases. In this study, all cases were unilocular. Cyst size in both previous reports and the present were as small as $\leq 4 \mathrm{~cm}$. Moreover, no relationship with basal cell nevus syndrome was 
seen in any cases in the present study. These results were consistent with those of the literature [4] [5] [6] [7].

Histopathologically, the average thickness of the lining epithelium was $4-8$ layers, with partial thickening observed in $17 \%$ of all cases [12]. Concerning rete ridges, $10 \%$ were flat and in contact with non-inflammatory connective tissue, and $23 \%$ were extended and showed contact with inflammatory granulation tissue [12]. These histopathological findings in the present study were consistent with previous findings [12]. Wright [12] described $2 \%$ of cases as showing no granular cell layer, whereas all cases were accompanied by that layer in the present study. Diagnostic evidence for OOC is a covering of orthokeratinized stratified squamous epithelium over the granular layer. Locally, OOC is attenuated keratinocyte by inflammatory change, it was described that must be merely local [12]. Stroma essentially comprised dense fibrous connective tissue, with variations in the degree of inflammation observed between cases. Concerning about the basal cell layer of OOC, cuboidal and squamous cells were often mixed [12], and this character was similar to the present results. Conversely, neoplastic characteristics of the basal cells are poor; i.e., no palisading and poor bud-like proliferation and daughter cyst formation [12] [23]. However, these findings were also observed in some cases in the present study.

Reports have described varying recurrence rates for OOC, of: 0.0\% [14] [15], $1.7 \%$ [12], 2.2\% [13], and 4.2\% [16], generally showing a low value. In the present study, the postoperative observation period was generally short and there was a limitation, but the recurrence rate was $0.0 \%$. Accordingly, treatment of OOC using cystectomy was not discussed. Mostcases of OOC have emerged as a painless swelling [13] [18], and many cases are discovered incidentally on $\mathrm{x}$-ray examination. OOC typically appears as a unilocular cyst accompanied by an impacted tooth in the half as dentigerous cyst (DC) [13] [18]. OOC shows a different biological attitude to $\mathrm{OKC}$ and is closer to DC rather than OKC, namely. Meanwhile, OOC shows a similar histology of epidermoid cysts accompanying the granular layer [12] [24] [25]. Besides, the pathogenesis is also referred to as aberrant ectodermal tissue due to trauma [26], but it is unclear. We thus surmised that OCC represents an independent concept as an odontogenic developmental cyst. However, Siar et al. [27] have reported squamous cell carcinoma accompanying OOC, facilitating early detection and appropriate treatment.

Although OOC and PC show similar predilections, discussion of the generation of both has been left. Li [23] described the origin of OOC as the tooth crest. However, Takeda et al. [28] have reported the presence of dermoid cyst arising from the jawbone. Consequently, basis for deciding on an odontogenic derivation for all jawbone cyst with orthokeratinized stratified squamous epithelium is insufficient. In addition, 2 cases of OOC that occurred in the gingiva have been reported previously [29] [30]. In the future, elucidation of the pathogenesis based on the accumulation of further cases and clarification of the molecular pathology will be needed. 


\section{Conclusions}

Within this limitation of this study, the following could be concluded:

1) OOC was occupied $2.1 \%$ of odontogenic developmental cysts with the mandibular molar area predominance in the present study.

2) Previous papers described that most were solitary, unilocular and related to impacted teeth.

3) OOC shows a different biological attitude to OKC and is closer to DC rather than $\mathrm{OKC}$.

\section{Acknowledgements}

This work was supported by JSPS KAKENHI Grant Number 18 K07000.

\section{Conflicts of Interest}

The authors declare no conflicts of interest regarding the publication of this paper.

\section{References}

[1] Hamilton, B.G.R. (1945) Classification of Cysts of the Jaws. The Journal of Oral Surgery, 31, 370-375. https://doi.org/10.1016/0096-6347(45)90114-1

[2] Philipsen, H.P. (2005) Keratocystic Odontogenic Tumour. In: Barnes, L., Eveson, J., Reichart, P. and Sidransky, D., Eds., Pathology and Genetics of Head and Neck Tumours, WHO Classification of Tumours, 3rd Edition, IARC Press, Lyon, 306-307.

[3] Speight, P., Fantasia, J.E. and Neville, B.W. (2017) Odontogenic and Non-Odontogenic Developmental Cysts. In: El-naggar, A.K., Chan, J.K.C., Grandis, J.R., Takata, T. and Slootweg, P.J., Eds., WHO Classification of Head and Neck Tumours, IARC Press, Lyon, 234-242.

[4] Simarpreet, V.S., Sudesh, K.R., Ramandeep, S.B. and Tushar, K. (2012) Orthokeratinized Odontogenic Cyst of the Mandible: A Case Report. International Journal of Oral \& Maxillofacial Pathology, 3, 69-73.

[5] Pillai, A.K., Gupta, M.K., Mhaske, S.J., Satpathy, M. and Singh, S.K. (2013) An Aggressive Orthokeratinized Odontogenic Cyst of the Mandible: A Case Report. IOSR Journal of Dental and Medical Sciences, 12, 45-48. https://doi.org/10.9790/0853-1214548

[6] Galván, M.C.G., García-García, A., Anitua-Aldecoa, E., Llamosas, R.M. and Aguirre-Urizar, J.M. (2013) Orthokeratinized Odontogenic Cyst: A Report of Three Clinical Cases. Case reports in Dentistry, 2013, Article ID: 67283.

https://doi.org/10.1155/2013/672383

[7] Pimpalkar, R.D., Barpande, S.R., Bhavthankar, J.D. and Mandale, M.S. (2014) Bilateral Orthokeratinized Odontogenic Cyst: A Rare Case Report and Review. Journal of Oral and Maxillofacial Pathology, 18, 262-266.

https://doi.org/10.4103/0973-029X.140776

[8] Sarvaiya, B., Vadera, H., Sharma, V., Bhad, K., Patel, Z. and Thakkar, M. (2014) Orthokeratinized Odontogenic Cyst of the Mandible: A Rare Case Report with a Systematic Review. Journal of International Society of Preventive and Community Dentistry, 4, 71-76. https://doi.org/10.4103/2231-0762.131265

[9] Tamura, N., Takeshima, H., Kikuchi, K., Goto, H., Aoki, R., Fujita, K., et al. (2015) 
A Case of Orthokeratinizing Odontogenic Cyst in the Mandible. The Journal of Meikai Dental Medicine, 44, 114-118.

[10] Shetty, D.C., Rathore, A.S., Jai, A., Thokchom, N. and Khurana, N. (2016) Orthokeratinized Odontogenic Cyst Masquerading as Dentigerous Cyst. International Journal of Applied Basic Medical Research, 6, 297-299. https://doi.org/10.4103/2229-516X.192597

[11] Mahdavi, N. and Taghavi, N. (2014) Orthokeratinized Odontogenic Cyst of the Maxilla: Report of a Case and Review of the Literature. Turkish Journal of Patholo$g y, 33,81-85$. https://doi.org/10.5146/tjpath.2014.01273

[12] Wright, J.M. (1981) The Odontogenic Keratocyst: Orthokeratinized Variant. Oral Surgery, Oral Medicine, and Oral Pathology, 51, 609-618.

https://doi.org/10.1016/S0030-4220(81)80011-4

[13] Crowley, T.E., Kaugars, G.E. and Gunsolley, J.C. (1992) Odontogenic Keratocysts: A Clinical and Histologic Comparison of the Parakeratin and Orthokeratin Variants. Journal of Oral and Maxillofacial Surgery, 50, 22-26. https://doi.org/10.1016/0278-2391(92)90187-5

[14] Iwabuchi, H., Yago, K., Tanaka, Y., Imatani, T., Honma, H., Susami, E., et al. (1996) Clinicopathological Variants of the Keratinization Type of Odontogenic Keratocyst. Japanese Journal of Oral and Maxillofacial Surgery, 42, 170-175. https://doi.org/10.5794/jjoms.42.170

[15] Mizuta, N., Kamimura, K., Nakano, H., Ota, Y., Iwai, S., Nakazawa, M., et al. (2008) Clinical Study of Keratocystic Odontogenic Tumor and Orthokeratinized Odontogenic Cyst. Journal of the Japanese Stomatological Society, 57, 267-271.

[16] MacDonald-Jankowski, D.S. and Li, T.K. (2010) Orthokeratinized Odontogenic Cyst in a Hong Kong Community: The Clinical and Radiological Features. Dentomaxillofacial Radiology, 39, 240-245. https://doi.org/10.1259/dmfr/36547074

[17] MacDonald-Jankowski, D.S. (2010) Orthokeratinized Odontogenic Cyst: A Systematic Review. Dentomaxillofacial Radiology, 39, 455-467.

https://doi.org/10.1259/dmfr/19728573

[18] Dong, Q., Pan, S., Sun, L.S. and Li, T.J. (2012) Orthokeratinized Odontogenic Cyst, a Clinicopathologic Study of 61 Cases. Archives of Pathology \& Laboratory Medicine, 134, 271-275.

[19] Vuhahula, E., Nikai, H., Ijuhin, N., Ogawa, I., Takata, T., Koseki, T. and Tanimoto, K. (1993) Jaw Cysts with Orthokeratinization: Analysis of 12 Cases. Journal of Oral Pathology \& Medicine, 22, 35-40. https://doi.org/10.1111/j.1600-0714.1993.tb00117.x

[20] Cohen, M.A. and Shear, M. (1980) Histological Comparison of Parakeratinised and Orthokeratinised Primordial Cysts (Keratocysts). The Journal of the Dental Association of South Africa, 35, 161-165.

[21] Neville, B.W., Damm, D.D., Allen, C.M. and Bouquot, J.E. (2009) Oral and Maxillofacoal Pathology. 3rd Edition, Saunders, Philadelphia.

[22] Siar, C.H. and Ng, K.H. (1988) Orthokeratinized Odontogenic Keratocysts in Malaysians. British Journal of Oral and Maxillofacial Surgery, 26, 215-220. https://doi.org/10.1016/0266-4356(88)90165-9

[23] Li, T., Kitano, M., Chen, X., Itoh, T., Kawashima, K., Sugihara, K., Nozoe, E. and Mimura, T. (1998) Orthokeratinized Odontogenic Cyst: A Clinicopathological and Immunocytochemical Study of 15 Cases. Histopathology, 32, 242-251. https://doi.org/10.1046/j.1365-2559.1998.00380.x 
[24] Toptas, O., Akkas, I., Tek, M., Ozan, F. and Boran, C. (2014) Intraosseous Epidermoid Cyst Associated with Impacted Mandibular Wisdom Teeth: An Uncommon Entity. Journal of Clinical and Diagnostic Research, 8, ZD31-ZD32. https://doi.org/10.7860/JCDR/2014/9413.4630

[25] Thomas, M., Tremain, R. and Laugharne, D. (2017) Epidermal Cyst of the Mandible: A Case Report. Oral Surgery, 10, 171-173. https://doi.org/10.1111/ors.12248

[26] Arora, A., Srivastava, D., Gupta, H., Kumar, V. and Kotwal, P.P. (2013) Sonographic Diagnosis of Subungual Intraosseous Epidermoid Cyst. Journal of Clinical Ultrasound, 41, 35-37. https://doi.org/10.1002/jcu.22058

[27] Siar, C.H. and Ng, K.H. (1987) Squamous Cell Carcinoma in an Orthokeratinized Odontogenic Keratocyst. International Journal of Oral and Maxillofacial Surgery, 16, 98-98. https://doi.org/10.1016/S0901-5027(87)80036-X

[28] Takeda, Y., Oikawa, Y., Satoh, M. and Nakamura, S. (2003) Latent Form of Multiple Dermoid Cysts in the Jawbone. Pathology International, 53, 786-789. https://doi.org/10.1046/j.1440-1827.2003.01560.x

[29] Chehade, A., Daley, T.D., Wysocki, G.P. and Miller, A.S. (1994) Peripheral Odontogenic Keratocyst. Oral Surgery, Oral Medicine, Oral Pathology, 77, 494-497. https://doi.org/10.1016/0030-4220(94)90229-1

[30] Boffano, P. and Gallesio, C. (2012) Peculiar Case of Orthokeratinised Odontogenic Cyst: A Peripheral Counterpart of the Intraosseous Entity? British Journal of Oral and Maxillofacial Surgery, 50, e75-e77. https://doi.org/10.1016/j.bjoms.2011.09.019 